\title{
Artigos
}

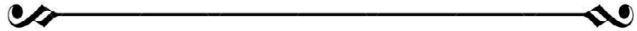

\section{EL PROCESO DE ELEGIBILIDAD EN ARGENTINA: RITUALES Y AMBIVALENCIAS EN EL RECONOCIMIENTO DE LOS REFUGIADOS}

\author{
The eligibility process in Argentina: rituals and ambivalences \\ in the recognition of refugees
}

Janneth Karime Clavijo*

\begin{abstract}
Resumen. La elegibilidad es uno de los procesos centrales en la construcción de las políticas de refugio y en la misma categoría de refugiado. Estos procesos remiten a instancias y mecanismos por medio de los cuales los Estados evalúan las solicitudes de asilo, lo que deriva en el reconocimiento y otorgamiento del Estatuto de refugiado o en el rechazo a dicha solicitud. En este artículo se examinan los discursos sobre los procesos de elegibilidad y el modo en que operan en la determinación de la condición de refugiado en el contexto argentino. El análisis se enmarca en los estudios que apuntan a revelar las dinámicas a través de las cuales se clasifican, y jerarquizan poblaciones. Se plantea que dentro de las categorías de solicitantes de asilo y refugiados es posible observar una heterogeneidad y ambivalencia en el modo en que son percibidos y clasificados los sujetos.
\end{abstract}

Palabras clave: refugio; elegibilidad; Argentina; solicitantes de asilo.

Abstract. Eligibility is a the central process in the construction of refugee policies and refugee status. These process refer to instances and mechanisms through which States evaluate asylum applications, which results in the recognition and granting of the Refugee Statute or rejection of such request. This paper examines the discourses about the eligibility process and the way these operate in the determination of refugee status in the Argentine context. The analysis is part of the studies that aim to visualize the dynamics through which populations are classified. Is proposed that within the categories of asylum seekers and refugees it is possible to observe a heterogeneity and ambivalence about the way in which subjects are perceived and classified.

Keywords: Refugee; eligibility; Argentina; asylum seekers.

\footnotetext{
* CIECS- CONICET y Universidad Nacional de Cordoba. Cordoba, Argentina.
} 


\section{Introducción $^{1}$}

En el marco de las migraciones catalogadas como forzadas, la cuestión del refugio ha devenido en uno de los temas prioritarios de las agendas internacionales. Si bien en el contexto reciente hay una proliferación de discursos y prácticas en torno a su tratamiento, la regulación del movimiento de refugiados ha sido una preocupación de larga data en el escenario internacional. Así, desde mediados del siglo XX se estableció la definición de la categoría de refugiado por medio de la Convención sobre el Estatuto de los Refugiados de 1951 y su Protocolo de $1967^{2}$, principales instrumentos internacionales en la materia, que fueron adoptados y reconfigurados en instancias tanto regionales como nacionales. En Sudamérica el surgimiento de la Declaración de Cartagena (1984) ha sido central, al establecer una definición ampliada de la categoría de refugiado ${ }^{3}$. A la vez, es posible observar un entramado de múltiples actores que han intervenido en la temática del refugio desde diversos ámbitos, entre los que se destaca la incidencia de los enfoques y prácticas promovidos por el Alto Comisionado de las Naciones Unidas para los Refugiados (ACNUR).

En las siguientes páginas analizo los discursos sobre los procedimientos y el modo en que operan en la evaluación y determinación de la condición de refugiado. Los procesos de elegibilidad remiten, a instancias y mecanismos por medio de los cuales los Estados evalúan las solicitudes de asilo, lo que deriva en el reconocimiento y otorgamiento del Estatuto de refugiado o en el rechazo a dicha solicitud. Mi indagación se enfoca en el modo en que estos procedimientos son desarrollados por parte de las instituciones en el contexto argentino, para lo cual enfatizo en los discursos y percepciones de los funcionarios. El presente trabajo se divide en cuatro partes, a lo largo de los primeros apartados hago referencia a los aspectos centrales

1 Las reflexiones planteadas en este texto se desprenden del análisis desarrollado en mi tesis doctoral titulada: "Políticas de refugio y asilo en Sudamérica: ambivalencias de la categoría de refugiado en la Argentina" (2017). Agradezco los comentarios y aportes constantes de mis directores Sandra Gil Araujo y Eduardo Domenech que nutrieron el trabajo. También agradezco a Andrés Pereira y Juan Carlos Sabogal por su lectura detallada.

2 Con la Convención de 1951, se establece la definición de refugiado referida a personas que: "debido a fundados temores de ser perseguida por motivos de raza, religión, nacionalidad, pertenencia a determinado grupo social u opiniones políticas, se encuentre fuera del país de su nacionalidad y no pueda o, a causa de dichos temores, no quiera acogerse a la protección de tal país" (ACNUR. Convención sobre el Estatuto de los Refugiados).

3 La definición ampliada establece que: "la definición o concepto de refugiado recomendable para su utilización en la región es aquella que además de contener los elementos de la Convención de 1951 y el Protocolo de 1967, considere también como refugiados a las personas que han huido de sus países porque su vida, seguridad o libertad han sido amenazadas por la violencia generalizada, la agresión extranjera, los conflictos internos, la violación masiva de los derechos humanos u otras circunstancias que hayan perturbado gravemente el orden público" (Declaración de Cartagena, p. 3. Disponible en: <http://www.acnur.org/5b076ef14.pdf>). 
en los procedimientos de elegibilidad, pasando de cuestiones generales a particularidades del ámbito nacional argentino, para luego, en los siguientes apartados, analizarlos bajo la noción de rituales estatales ${ }^{4}$, y profundizar sobre elementos vinculados a las situaciones de entrevista, por último, recapitulo las ideas principales y propongo algunas consideraciones finales. A lo largo de este artículo, planteo que dentro de las categorías de solicitantes de asilo y refugiados es posible observar una heterogeneidad y ambivalencia en cuanto al modo en que son percibidos y clasificados los sujetos, lo que revela un abanico de representaciones que emergen en los discursos institucionales.

Indagar sobre las representaciones institucionales también implica prestar atención a las características y conductas que idealmente les son atribuidas a los merecedores del Estatuto de refugiado. El análisis pone en evidencia el modo en que las poblaciones son diferenciadas, desde el ámbito normativo y de la intervención, a partir de la asignación de ciertas categorías 5 . Una de las características centrales de la temática del refugio es la dimensión moral ${ }^{6}$, como un hilo transversal y a la vez dinámico, desde el cual se han promovido diversas prácticas. Es decir se aplican diferentes etiquetas ${ }^{7}$ sobre un mismo sujeto y un mismo proceso de movilidad, con implicancias diversas en las condiciones de vida en el lugar de destino.

Parto de la premisa que la construcción de los criterios clasificatorios en el marco de los procesos migratorios está imbricada con el orden nacional, por tanto una de las primeras distinciones que produce la organización del mundo en Estados-nacionales es la diferenciación entre nacionales y no-nacionales ${ }^{8}$. Así, las diferentes categorías que se han construido para ordenar las migraciones,

4 Inspirada en la propuesta sobre rito de institución que plantea Bourdieu como "acto solemne de categorización que tiende a producir lo que designa". Es un acto de autoridad que expresa y significa al otro, es decir que construye aquello que aparentemente solo describe (Bourdieu, 1985, p. 80).

5 Atendiendo a las discusiones sobre las clasificaciones de las migraciones que han planteado Gil Araujo (2010) y Domenech (2013); y sobre la cuestión del refugio Zolberg (1983) y Malkki (1992).

6 Se contemplaron como de antecedentes autores que abordan esa dimensión moral y emotiva, entre ellos: Fassin (2016); Kobelinsky (2013).

7 La noción de etiquetas retomada de Zetter apunta a reflexionar sobre el proceso de categorización de las migraciones forzadas (desplazado forzado, refugiado, refugiado ambiental, voluntario/ no voluntario, entre otras). Desde esta mirada se propone observar el proceso mediante el cual el poder clasificatorio de las políticas de refugio categoriza a los sujetos. En el marco de sus análisis de los movimientos masivos de refugiados, Zetter señala la idea de etiquetamiento (labelling) vinculada a los procedimientos y prácticas burocráticas que configuraban las identidades de los refugiados, como también, a las formas en que se fracciona la etiqueta del refugiado para garantizar la gestión y administración de procesos migratorios globales, en especial las denominadas migraciones forzadas (Zetter, 2007).

8 Siguiendo a Sayad el orden nacional y el orden de las migraciones "están consubstancialmente ligados el uno con el otro"; por tanto las poblaciones son reconocidas y ordenadas como poblaciones nacionales (Sayad, 2008, p. 102). 
revelan los modos en que una presencia no-nacional es definida como legítima o ilegítima .

Este trabajo se llevó a cabo a partir de una metodología cualitativa, basada en el análisis de un corpus que contempla diferentes documentos producidos por variados actores; $y$ entrevistas semiestructuradas a funcionarios públicos ${ }^{10}$, en particular de la Comisión Nacional para los Refugiados (CONARE). Cabe señalar, que a lo largo de este trabajo, acudí a una mirada que aborda epistemológicamente los discursos y representaciones en el marco de las políticas como objetos de indagación (Shore, Wright, 1997).

\section{El proceso de elegibilidad: ¿el ritual que performa?}

En este apartado, me propongo dar cuenta de algunos elementos primordiales en los procesos de elegibilidad. A la vez, pondré de relieve cuestiones que se presentan como dilemáticas y que permiten problematizar esta instancia que atraviesan los solicitantes de asilo. El denominado proceso de elegibilidad pone en evidencia la coexistencia del derecho de las personas a solicitar asilo y la potestad soberana de los Estados en el reconocimiento (o no) de la condición de refugiado. Si bien desde la Convención de 1951 existen directrices establecidas en instancias internacionales y regionales a las cuales la mayoría de los Estados Sudamericanos han adherido, pensar en los procesos de elegibilidad nos remite al ámbito nacional, aunque estén atravesados por lineamientos del plano intergubernamental, la autorización o no de la permanencia en el territorio nacional también depende de las decisiones que se adopten en el marco de las políticas al interior de los Estados y de la forma en que se configuren prácticas como los procesos de elegibilidad.

Por otra parte, el ACNUR ha tenido un papel central en la construcción de las directrices para la interpretación e intervención de la temática del refugio. En ese sentido, propuso que en el marco de los sistemas de ingreso a los Estados, además de los funcionarios de ingreso que tienen el primer contacto, sean considerados como guardianes de los procedimientos todos aquellos agentes estatales que, de un modo u otro, intervienen en el contacto

9 La figura de refugiado se encuentra asociada a un sujeto que carece de la protección de un Estado que garantice su vida y sus derechos, lo que implica que provisoriamente otro Estado asuma dicha garantía en el marco de la protección internacional humanitaria.

${ }^{10}$ El corpus de la investigación doctoral de la cual se desprende este artículo contempló además de los diversos documentos recopilados quince entrevistas semiestructuradas, las cuales fueron llevadas a cabo entre los años 2014 y 2015. Los entrevistados fueron funcionarios públicos vinculados a instituciones estatales involucradas en la formulación e implementación de las políticas de refugio, como también a representantes del ACNUR y de organizaciones no gubernamentales. En la sistematización del corpus utilicé el programa de análisis cualitativo de datos Atlas-ti, por medio del cual codifiqué, organicé y advertí elementos trasversales en el marco del tratamiento de la cuestión del refugio. Cabe señalar, que en este texto retomo en particular extractos de las entrevistas que hacen referencia a la elegibilidad en el marco del reconocimiento de la solicitud de asilo. 
con los sujetos. Ese proceso de selección ha sido protocolizado para que, a modo de buena práctica, se replique por los diferentes agentes estatales, lo que supone unificar una forma de llevar a cabo los procedimientos, y establecer una mirada al menos compartida, sobre la asignación de una categoría a partir de determinadas características e intereses de los Estados y organismos involucrados.

La construcción y adopción de esas buenas prácticas no necesariamente es conjunta y consensuada, dadas las relaciones jerárquicas que atraviesan la construcción de estos marcos políticos internacionales; como tampoco es uniforme y del todo predecible el modo en que se llevan a cabo en los diversos contextos, más allá de lo estipulado en documentos. A esta primera línea de funcionarios que intervienen en el proceso de evaluación se suman los oficiales de elegibilidad, de los que se presume serán los sujetos con mayor formación e idoneidad para atender el caso a caso de las solicitudes. En Argentina estos funcionarios dependen directamente de la Comisión Nacional para los Refugiados (CONARE), y son los que realizan las entrevistas, construyen el expediente de cada solicitud, y con base en ello recomiendan a la Comisión otorgar o no el estatuto como refugiado, por lo tanto son funcionarios centrales en el proceso de validación de la solicitud.

De acuerdo con los documentos del ACNUR, los diversos guardianes del procedimiento estarían encargados de recolectar la información primaria y secundaria para la evaluación de las solicitudes. La recolección de dicha información es concebida como una estrategia para construir múltiples perfiles. Estos perfiles preliminares se enfocarían en la identificación de ciertas demandas urgentes, y en establecer un primer ordenamiento de los sujetos con base en los criterios preestablecidos. Se advierte que esa primera clasificación puede ser errónea y por lo tanto sujeta a cambios a largo del proceso (ACNUR, 2009, p. 136).

Si bien se reconoce la dificultad en el proceso de identificación, la práctica en sí misma no es cuestionada, es decir, se sostiene la necesidad de clasificación y la asignación de una u otra categoría como mecanismo necesario para la atención a los sujetos y para la gestión de los procesos migratorios, lo que implica que aunque se reconozcan el cruce varias situaciones y demandas, se prioricen solo algunas de ellas. En ese caso, la posibilidad de auto-identificación de los sujetos está mediada por las consideraciones y la validación de los funcionarios en destino, lo que reduce el margen de autonomía y revela la demanda por organizar y administrar a la población, en particular la población no-nacional, tanto de los Estados como de los organismos involucrados, conjugada con intereses que van más allá de las complejas situaciones de los sujetos. En ese sentido, el ACNUR también aclara que en la instancia de identificación de perfiles deben intervenir diversos 
organismos, ya que dependiendo de la categoría asignada a los sujetos se debe referir el caso a uno u otro ente institucional, dada la presencia de sujetos que no son contemplados dentro del plano de la protección, como los llamados migrantes económicos (ACNUR, 2009).

Es decir, conviven junto con la preocupación por la atención a los sujetos la mirada selectiva y una concepción e intervención segmentada sobre los mismos procesos y sujetos migrantes. Esa mirada y concepción se encuentra marcada por múltiples dimensiones. En ese sentido, más allá de las herramientas y mecanismos a desarrollar que enuncian los marcos normativos y procedimentales, estos están encarnados en el día a día de las prácticas institucionales, donde la subjetividad de los funcionarios expresada en diversas formas atraviesa esa categorización ideal y es un factor clave para comprender el tratamiento sobre la cuestión del refugio.

\section{La elegibilidad en el escenario nacional: actores y procedimientos}

En el ámbito nacional, la CONARE ${ }^{11}$ es el organismo en el que se llevan adelante los procesos de elegibilidad. Esta tarea, aunque se desarrolla en cada Estado, se vincula con la necesidad de corroborar que la situación de los solicitantes de asilo corresponda con la definición formal de refugiado, y que no haya causas por las que no debiera concederse la protección. Ahora bien, la CONARE como Comisión Interministerial está compuesta por representantes del Ministerio del Interior, Ministerio de Justicia y Derecho Humanos, Ministerio de desarrollo Social, la Cancillería, y el Instituto Nacional contra la Discriminación, Xenofobia y Racismo (INADI) ${ }^{12}$; además, cuenta con la participación con voz, pero sin voto, del ACNUR y de una organización de la sociedad civil vinculada en sus labores con el tema, y cuya elección se renueva cada dos años. Al momento de indagación de este trabajo (20142015), la institución que ocupaba este lugar era la Fundación Comisión Católica Argentina de las Migraciones (FCCAM) ${ }^{13}$.

En cuanto al desarrollo del procedimiento de elegibilidad, en primer lugar, se plantea que cualquier persona puede solicitar asilo directamente en la oficina de la CONARE, o en los puestos fronterizos, o cualquier lugar donde tengan presencia autoridades argentinas, gendarmería, prefectura, o

${ }^{11}$ Esta instancia surge con la aprobación Ley $N^{\circ} 26.165$ de Reconocimiento y Protección al Refugiado en el año 2006, en reemplazo de la Comisión de Elegibilidad para los Refugiados (CEPARE) que fue establecida en 1985.

${ }^{12}$ El INADI es un organismo descentralizado creado en 1995, se ubica en la órbita del Ministerio de Justicia y Derecho Humanos de la Nación. Sus labores incluyen la elaboración de políticas nacionales para combatir toda forma de discriminación, xenofobia y racismo.

${ }^{13}$ La FCCAM es una organización de la sociedad civil que a partir de sus labores se orienta a la sensibilización de la comunidad sobre el fenómeno de las migraciones y su incidencia en la sociedad. 
delegaciones de la Dirección Nacional de Migraciones (DNM) ${ }^{14}$. Sin embargo, es en las instalaciones de la CONARE en la ciudad de Buenos Aires donde se presenta el mayor número de solicitudes.

Esa presentación es realizada en forma escrita, a través del formulario de solicitud de reconocimiento de la condición de refugiado que es provisto en las distintas instalaciones a las cuales los sujetos podrían acudir. En los primeros párrafos del formulario se informa al solicitante que bajo la vigencia del procedimiento no será devuelto al país origen, que se le otorgará un documento en el cual se explicita que puede permanecer en el país hasta que sea resuelto el caso en forma definitiva.

A continuación se incluyen varios ítems relacionados con el carácter confidencial de la solicitud; y el compromiso de cooperar diciendo la verdad y aportando toda prueba e información que corrobore los hechos expuestos, así como también la obligación de concurrir a las instituciones para renovar el documento de residencia precaria previo a su vencimiento.

En una segunda parte del formulario, se plantean las preguntas iniciales asociadas a la definición de la categoría, las motivaciones y argumentos que fundamentan la solicitud, en particular las formas de desplazamiento y estrategias de huida interna en el país de origen, hasta la elección de Argentina como lugar de destino y la forma de ingreso al país. Con relación al funcionamiento de las labores de elegibilidad, los funcionarios plantean algunos cambios en la estructura de la CONARE que procuraron unificar criterios para abordar y resolver los casos. Se aclara que:

Antes los oficiales de elegibilidad trabajaban como muy independientemente $y$ hace un tiempo empezamos como primero a nombrar un coordinador, que supervisa y lee todo entonces el criterio se unifica, porque, nos pasaba que la CONARE nos dijera, a ver este caso lo entrevistó Juan y lo resolvió negativo, mientras que este caso es muy parecido y lo entrevistó Pedro y lo resolvió positivo, y eso no puede suceder. ${ }^{15}$

Con respecto a la resolución de los casos, el mecanismo estipulado para la determinación de la condición de refugiado, una vez realizadas las entrevistas y construido el informe del caso, consiste en la deliberación y votación de los comisionados, considerando el expediente previo presentado por los funcionarios de elegibilidad ${ }^{16}$.

\footnotetext{
${ }^{14}$ En esos casos, se plantea que, ante la presentación de una solicitud de asilo desde las delegaciones de la DNM se establece contacto con funcionarios de la CONARE, e incluso cuando es requerido los funcionarios de elegibilidad se desplazan a las diferentes provincias.

${ }^{15}$ María. Funcionaria de la CONARE. 2014.

${ }^{16}$ El reglamento interno de la CONARE explicita: La Comisión sólo podrá adoptar decisiones en lo referente a las cuestiones de su competencia, sobre el estatuto de refugiado de una persona en
} 
Los funcionarios también plantean los tiempos de resolución de solicitudes como una de las preocupaciones centrales en el sistema de asilo en Argentina. Esta condición, que se revela como una inquietud en torno al funcionamiento eficiente de la CONARE, también implica la prolongación en el tiempo de la condición de solicitantes de asilo, lo que supone posiciones ambivalentes entre el reconocimiento de la condición como refugiado y el rechazo a la solicitud. Esto deriva, como veremos, en la posibilidad de ser interpelados desde otras categorías vinculadas a la migración económica y a otras condiciones de tratamiento. En palabras de una funcionaria de la CONARE:

Nos falta un montón de cosas por mejorar, por ejemplo siempre el ACNUR elogia como los buenos estándares que tiene la Argentina, pero pienso que eso muchas veces ha atentado contra la celeridad en las resoluciones, que es nuestro gran tema, nosotros tenemos una capacidad para resolver casos que es limitada, y en general demoras, depende de la complejidad del caso, pero en promedio habrán 9 meses de demora en la resolución de caso y me parece que es demasiado y hay que bajarlo, tal vez porque los informes son largos, son complejos, se discuten. ${ }^{17}$

Por otra parte, los procedimientos que se contemplan en el marco de la elegibilidad han revelado cambios en su implementación, en Argentina se han establecido procedimientos diferenciados para la resolución de las solicitudes, los cuales están dirigidos hacia ciertos casos cuya respuesta se hace de forma más expeditiva. El ACNUR, ha sido promotor de la implementación de estas propuestas y ha venido insistiendo en la adopción de algunas herramientas para la gestión eficiente de las solicitudes que tienen que ver con el mecanismo de examen de la solicitud, básicamente referidas a procedimientos de admisibilidad y procedimientos acelerados, algunos documentos explicitan que: "el ACNUR sí reconoce que los procedimientos nacionales pueden incluir disposiciones especiales para el manejo expeditivo de solicitudes que claramente carecen de fundamento y que no ameritan un análisis completo en cada etapa del procedimiento" (ACNUR, 2000, p. 11).

Para el caso argentino, y en armonización con lo que planteó el organismo internacional, los procedimientos diferenciados, que implican una atención prioritaria en la resolución de la solicitud, son llamados procedimientos sumarios y suponen que la CONARE debería pronunciarse en un periodo menor a 6 meses. Diferenciados del procedimiento ordinario, que remite a procesos de indagación más largos a los solicitante de asilo. Al respecto, un funcionario de la CONARE comenta:

el país, por la mayoría simple de la Comisión reunida en quórum. En caso de empate el voto del Presidente se computará doble. CONARE. Reglamento Interno.

17 María. Funcionaria de la CONARE. 2014. 
Tenemos otro tipo de procedimiento que se llama sumario, un procedimiento acelerado, que es para los casos que consideramos manifiestamente fundados, que verdaderamente tienen una necesidad imperiosa de protección, y para los casos que también consideramos como manifiestamente infundados, casos que no requieren una necesidad de protección. ${ }^{18}$

La adopción de estos procedimientos está constantemente asociada a la lectura sobre las causas del desplazamiento que expresen los sujetos, con especial importancia en la distinción entre los verdaderos y falsos refugiados, a partir de cómo es fundado o no el hecho de recurrir a la solicitud, aquello que en el momento de la solicitud es considerado como una causa válida y qué se pone de relieve sobre otras motivaciones para que los sujetos puedan ser leídos como legítimos refugiados, ligado las interpretaciones de los funcionarios, a la luz de las definiciones de la categoría. Como en las narraciones, en las interpretaciones se entrelazan diversos elementos, incluso a veces van de la mano de generalizaciones adjudicadas a partir del origen nacional,

En el año 2009 cuando se empezaron a suceder solicitudes de dominicanos y de senegaleses, se llegó a las 1400 solicitudes en un año, y la verdad es que la mayoría eran solicitudes que se podían catalogar como manifiestamente infundadas, solicitudes que era claro que no tenían ninguna vinculación con la Convención, los senegaleses sobre todo decían 'he venido por razones económicas, porque no tenía trabajo', pero es cierto que su derecho a peticionar lo tenían y lo que dijimos es, no está mal establecer un procedimiento con todas las garantías, pero más acelerado para resolver estas solicitudes, como separar los casos complicados y los casos que son sencillos, ya sea para la denegatoria y para el reconocimiento, ideamos un procedimiento que fue la primera resolución que tomó la CONARE, el acta 1 de la CONARE, un procedimiento sumario. ${ }^{19}$

Varias cuestiones emergen de este fragmento. En primer lugar, la desestimación de la solicitud en tanto se fundamente o se interprete como motivada bajo la necesidad de inserción laboral pone en evidencia la idea de entender la migración forzada desligada de la dimensión económica. Más aún, el verdadero refugiado aparece asociado a una víctima cuyas posibilidades y estrategias de supervivencia se encuentran en el plano de la asistencia (al menos en esta instancia), es decir, cuando los sujetos plantean el acceso al trabajo como una de sus preocupaciones no se condicen con las representaciones que circulan sobre alguien que requiere protección como refugiado, aunque sea un aspecto presente en la vida de todos los sujetos, y no excluyente de otras preocupaciones.

\footnotetext{
${ }_{18}$ Jorge. Funcionario de la CONARE. 2014.

${ }^{19}$ María. Funcionaria de la CONARE. 2014.
} 
Cabe agregar que en el discurso de los funcionarios se plantean algunos factores que han incidido en el aumento de solicitudes de asilo, en particular aquellas consideradas manifiestamente infundadas al estimar que la motivación para presentar la solicitud se relaciona con la búsqueda de la regularización migratoria (Nejamkis, Álvarez, 2012). Así, se señala que los migrantes recurren al sistema de asilo cuando no reúnen los criterios migratorios estipulados en la Ley migratoria (en particular asociado a casos de senegaleses y dominicanos), aquellos que "encuadran en el criterio" migratorio pero no reúnen los requisitos para la regularización por haber ingresado al territorio de forma "ilegal" y la facilidad, en el caso de presentar la solicitud de asilo, en no presentar documentación (Nejamkis, Álvarez, 2012).

Lo manifiestamente fundado en el marco del refugio supone otra performance, acentuar aspectos como el temor que generen una correspondencia con la categoría a juicio del funcionario de elegibilidad, y sobre los cuales éste (el funcionario) justifica invocar la solicitud. En ese sentido, la idea de casos sencillos parece estar relacionada con la contundencia con la que estas representaciones son construidas en el momento inicial del procedimiento, en algunos casos asociadas a generalizaciones desde las cuales se abordan varias solicitudes, como por ejemplo un mismo origen nacional.

A la par, los casos que son interpretados como manifiestamente infundados también son adjetivados como abusivos o fraudulentos, bajo la concepción de que la categoría de refugiado alude a un sujeto merecedor de esa mirada compasiva desde la cual se interpela a los Estados como protectores, y el recurrir a este amparo de manera infundada es leído como un abuso del cual debe ser cuidado el mismo estatuto y el Estado. En esa dirección se argumenta la existencia desde la CONARE de:

procedimientos diferenciados para las solicitudes manifiestamente infundadas, abusivas o fraudulentas, y para las manifiestamente fundadas, por ejemplo el caso de los sirios son manifiestamente fundadas, requieren una protección que es evidente, hoy se resuelven con un procedimiento diferenciado y acelerado de forma mucho más rápida, y por otro lado, aquellos que vienen y dicen 'no en realidad, voy a trabajar y necesito la precaria para poder trabajar, pero no tengo miedo', bueno en ese caso se resuelve o se trabaja en un procedimiento también acelerado y diferenciado porque es manifiestamente infundado. ${ }^{20}$

La instauración de estos procedimientos diferenciados también supone una alternativa para descomprimir la demanda burocrática en el sistema de asilo. Sin embargo, no es problematizada la naturaleza selectiva y el imperativo de catalogar sobre el que se asientan las políticas de refugio y, en general, las políticas hacia los procesos de movilidad humana, por lo que tampoco

${ }^{20}$ Federico, A. Presidente de la CONARE. 2014. 
se traduce en una respuesta diferenciada con base en las prioridades de las personas, quienes ante la denegatoria quedan en un limbo sobre sus posibilidades y condiciones de permanencia, en muchos casos en situación administrativa irregular, con todos los efectos que ello implica en su vida cotidiana, entre ellos la posibilidad de expulsión. Algunos funcionarios frente a la denegatoria de la solicitud por procedimiento sumario comentan: "Ios que quedaron van al tratamiento ordinario, donde se hace la entrevista, lo sumario es como bueno sos un migrante aquí, ojala que arregles y sobrevivas" 21 .

La incertidumbre y ambivalencia que aparece en el transito como solicitante de asilo se expresa también en la residencia precaria como forma constante de revalidación de su presencia, durante el tiempo que lleve la evaluación. Esto lo puede derivar, en caso de rechazo de la solicitud, al plano exclusivamente migratorio, desde el cual los criterios frente a su permanencia se asocian a otras dimensiones, se aclara que "una vez que ya es rechazada su apelación, ya no es más solicitante tampoco, ya se le dice bueno mirá por este camino no, tienes que ser un migrante común y corriente, y fijate de regularizar tu situación como un migrante" ${ }^{22}$.

Las citas anteriores evidencian tanto, la ambivalencia que opera en el ejercicio de categorización de los sujetos, como las formas en que la misma institución configura los sujetos de su intervención y erige los límites de su competencia en materia de protección a otros funcionarios. A la par, en el marco de las políticas de refugio, es posible considerar la construcción de procedimientos orientados a la instrumentalización de fibras propias del plano emocional y moral ${ }^{23}$. El sistema de asilo se sustenta en el temor fundado como emoción que legitima la presencia de los refugiados, e intenta objetivar esa emoción a partir de la adopción de criterios normativos y mecanismos estandarizados considerados efectivos para el ejercicio de la categorización.

\section{La entrevista: ¿encuentro para el discernimiento?}

Sin duda, las situaciones de entrevista son uno de los pasos centrales de la elegibilidad, por ello desde las instituciones se hace énfasis sobre quiénes las realizan y cómo se desarrollan. Más allá del modo en que son planteadas, es posible observar que implican un escenario donde el encuentro personal con el solicitante está atravesado por el mismo espacio estatal. La posición desde la cual parte el entrevistador establece una relación asimétrica entre los sujetos presentes (funcionario y solicitante), donde la exposición del relato del solicitante de asilo estará evaluada bajo la lupa del funcionario de elegibilidad

\footnotetext{
21 Violeta. Comisionada de la CONARE. 2014.

22 Juliana. Funcionaria de la FCCAM. 2014.

${ }^{23}$ Sobre este punto ver KOBELINSKY, 2013.
} 
quien, además de su trayectoria, interpretaciones y (pre)juicios personales, se encuentra investido por la discrecionalidad y el aura estatal (Taussig, 1998).

A partir de los comentarios que los funcionarios compartieron sobre sus experiencias, es posible develar la emergencia de la ambivalencia en torno a los modos en que se entretejen, en la entrevista, criterios que van desde asumir el encuentro como un procedimiento técnico hasta las ebullición de impresiones subjetivas latentes e inevitables a partir de la misma condición humana de los funcionarios de elegibilidad, entrelazadas con su lugar como nacionales, agentes estatales, y evaluadores del temor y la verdad del otro solicitante de asilo, no-nacional. En otras palabras,

La verdad no es sencillo, el análisis que uno hace es desde la posición de la clase media porteña y es muy difícil ponerse en el rol de la persona que te está narrando que iban a someterlo a un sacrificio humano en Africa, por poner un ejemplo, y son las cosas que uno dice iserá cierto? de entrada es como tan raro, a nosotros siempre en los cursos se nos dice que lo primero que hay que hacer es quitarse todos esos prejuicios de encima. ${ }^{24}$

Esta ambivalencia y tensión entre lo técnico y lo subjetivo emerge como una constante en las labores de quienes desarrollan los procedimientos de elegibilidad. Sus tareas atraviesan otros planos vinculados con condiciones emocionales de los mismos funcionarios, trabajadores humanitarios como ellos mismos se nominan,

La verdad es que uno está acá como cotidianamente leyendo los casos, en líneas generales de 10, 9 son casos sencillos, pero siempre hay uno que afecta, todos los que trabajamos acá alguna historia te pueden contar que tuvieron un momento de esos de despertarse de noche, pensando en el relato. ${ }^{25}$

La manera en que se cruzan diferentes elementos en los discursos de los funcionarios en la posición de entrevistador parece una interpelación a la propia práctica. Sin embargo, esa autoreferencia en un contexto que se percibe como diferente de aquel que llega, y atravesado a la vez por dimensiones como clase, nacionalidad, el rol como funcionario, ubica al entrevistador en una situación de detentor de la nominación (Bourdieu, 1997), en tanto es quien, como agente estatal a través de los procedimientos adoptados, decide y clasifica al solicitante. De alguna manera la entrevista es un lugar donde esos marcos normativos ideales se encarnan y revelan su ambigüedad, es en el oficio del funcionario de elegibilidad donde también se construye, se ejerce y se reproduce la clasificación estatal, es el reflejo del Estado en acción en dicha práctica de selección.

\footnotetext{
${ }^{24}$ María. Funcionaria de la CONARE. 2014.

${ }^{25}$ María. Funcionaria de la CONARE. 2014.
} 
De hecho, varias de las guías difundidas por el ACNUR plantean las actitudes y comportamientos como parte de la evaluación de la verdad a partir del encuentro con los solicitantes.

La audiencia personal es sumamente importante debido a la dificultad para determinar la credibilidad del solicitante sólo con base en la transcripción de una entrevista o un informe. Una audiencia personal permite evaluar la actitud y el comportamiento del solicitante de asilo y formular preguntas adicionales y detalladas. (ACNUR, 2001, p. 59)

Vinculado a la situación de entrevista, en varios documentos, incluyendo la Ley General de Reconocimiento y Protección de Refugiados 26.165 de 2006, se plantean ciertas obligaciones para el solicitante desde las cuales es posible perfilar las características y el comportamiento esperado de un solicitante genuino. Entre ellas: decir la verdad y ayudar a esclarecer los hechos invocados y los motivos personales en que se basa su solicitud; esforzarse por aportar pruebas y suministrar explicaciones satisfactorias; proporcionar información personal sobre su experiencia con los detalles necesarios para determinar los hechos pertinentes; contestar a todas las preguntas que se le formulen ${ }^{26}$.

Desde esa perspectiva se concibe la verdad como un hecho objetivo en tanto los sujetos deben dar cuenta en su relato, de coincidencias en el orden del discurso, que permitan al entrevistador aseverar, además de la factibilidad de esa narración, una coherencia intrínseca de los hechos expresados. En ese sentido, la verdad aparece cuando ese discurso, tanto en su dimensión verbal como no verbal, es considerado verídico y conmovedor a los ojos del funcionario de elegibilidad:

Los chicos se indignan, porque dicen yo lo tuve adelante y estoy convencido, siempre se dice la credibilidad no tiene que ver con la persona, y con la sensación que me produjo, sino con que su relato sea coherente, que lo que diga se condice con la información en el país de origen, que no haya mayores contradicciones, son como indicadores objetivos, pero también hay una cuestión de feeling, no sé cómo Ilamarlo. ${ }^{27}$

Es posible reconocer también una posición dicotómica en el funcionario de elegibilidad, con un marcado acento en los criterios objetivos y a la vez una dimensión personal evidente e indivisible. En el proceso de elegibilidad concebido como un procedimiento objetivo y técnico se invisibiliza o se intenta trasladar a ese plano una evaluación que es básicamente moral y emocional, mediada por posiciones de los sujetos en el marco de la estructura estatal, que de entrada supone relaciones asimétricas. Son dos personas en

\footnotetext{
${ }^{26}$ Ley General de Reconocimiento y Protección de Refugiados 26.165 de 2006. Art. 44.

${ }^{27}$ María. Funcionaria de la CONARE. 2014.
} 
posiciones desiguales: una sometiéndose a la evaluación y exponiendo su relato, y otra evaluando la veracidad de lo que expresa. A la vez, en la misma estructura del organismo la dinámica también supone relaciones jerárquicas entre los funcionarios, donde, más allá de las apreciaciones en el informe de elegibilidad, los Comisionados son quienes ratifican en última instancia la decisión. Allí la valoración del denominado temor fundado aparece como elemento esencial, en tanto eje de los criterios que mediaran el juicio sobre las decisiones y las trayectorias asumidas por los sujetos.

A veces no es sencillo, a veces lo que nos pasa además es que el oficial que toma el caso, se compromete con el caso porque tuvo a la persona enfrente, comprendió la lógica del relato, registró la entrevista, eso llega a manos de la CONARE y capaz que considera que no es un caso creíble. $^{28}$

El temor fundado y la verdad suponen no solo que las narraciones y los comportamientos de los sujetos correspondan a los criterios y definiciones de refugiado, sino que además se haya transitado por ciertas reacciones previas, como búsqueda de protección en el país de origen antes del cruce de las fronteras nacionales. En ese sentido, las entrevistas personales también se evidencian como instancias de convencimiento y justificación.

Así, el proceso de elegibilidad escudriña y pone de relieve ciertos aspectos de los solicitantes sobre otros, implica un performance de lo que supone el verdadero refugiado, que reduce y esencializa a la personas a esos aspectos, como el temor fundado o la condición de víctima, de modo que dependiendo de lo que se priorice se habilita o no el reconocimiento.

Por otro lado, las estrategias que despliegan los solicitantes no necesariamente cuestionan los procedimientos estatales. En este sentido, si bien el modo en que se expresa ese orden nacional está marcado por las particularidades de cada Estado-nación, el pensamiento de Estado (Sayad, 2010) es una matriz que compartimos en un mundo organizado por Estados. Ese pensamiento se encarna a nivel micro en las prácticas de los sujetos, no solo en los funcionarios de elegibilidad, sino en los solicitantes que atraviesan el proceso de evaluación. De este modo, también hay una validación y participación, aunque en una posición asimétrica, de los sujetos en lo que se podría llamar el ritual estatal de elegibilidad ${ }^{29}$ como forma de acceso y

${ }^{28}$ María. Funcionaria de la CONARE. 2014.

${ }^{29}$ Como señalé en las páginas iniciales la noción de ritual estatal está inspirada en la idea de rito de institución que desarrolla Bourdieu. En este caso, enfocada en los procedimientos estatales de elegibilidad, y teniendo en cuenta la capacidad de producir e imponer categorías de pensamiento como un elemento clave para comprender el modo en que opera y se reproduce el orden estatal, y las maneras en que, a través de discursos y prácticas aparentemente objetivas, se construyen "estructuras mentales, formas de percepción y de pensamiento" (Bourdieu, 1997, p. 91). 
legitimación de la permanencia en un territorio nacional bajo el manto de la protección, sea lo que sea que esta implique, y más allá del uso estratégico o la identificación que pueda generar con esas categorías.

Es significativo considerar que las atribuciones de poder son diferentes y que eso puede implicar, en muchos casos, que las personas se encuentren en situaciones condicionantes desde las cuales vivenciar estos procedimientos. El poder estatal, atravesado por los procesos técnico-burocráticos, coloca en un lugar diferenciado al funcionario (nacional), posición que en apariencia supone un efecto de neutralidad en sus juicios subjetivos, frente a la evaluación de las experiencias, emociones y vivencias del otro, solicitante de asilo (nonacional); el ritual funciona en la medida en que ambos se reconocen en esas posiciones. La cosmovisión desde la mirada estatal no es cuestionada; al igual que cualquier proceso naturalizado es un acto de fe en el que tanto unos como otros son participes.

En las entrevistas a los funcionarios podemos identificar, dos cuestiones: en primer lugar un lenguaje específico que apela a ciertas construcciones normativas y técnicas para avalar definiciones, limitaciones y márgenes de quién podría ser o no un verdadero refugiado; y en segundo lugar, en relación con lo anterior, una mirada más emotiva y moral, que estipula, en planos más dicotómicos, cuestiones vinculadas a valores como verdad, temor fundado, engaño, etc., y que revela lógicas particulares de dimensionar las clasificaciones.

De manera que la misión de los oficiales de elegibilidad remite a quiénes pueden discernir, y sugerir otorgar o no el estatuto. A la vez, la discrecionalidad del oficial de elegibilidad como guardián del procedimiento puede ser apelada por otras discrecionalidades de funcionarios de mayor rango, quienes, investidos de un poder particularizado en la estructura burocrática, toman las decisiones en el ámbito de la CONARE. En este sentido, lecturas particulares de la realidad son construidas por agentes estatales que reinterpretan la normativa y las narrativas de los solicitantes. Además, por medio de discursos sobre la figura del refugio, constituyen regímenes de verdad y refugiado hiperreal (Mc Callum, 2012).

En este marco, y partiendo de la idea de pensar la elegibilidad como un ritual estatal, lo que permite distanciar la mirada de un plano meramente normativo, cabe preguntarnos por la construcción misma de esos rituales estatales (siempre dinámicos), presentes en los procesos en los que se asigna una u otra categoría a los sujetos ${ }^{30}$. Pensar los rituales como parte de los

\footnotetext{
${ }^{30}$ Cabe señalar que el modo en que la elegibilidad opera en el acceso al estatuto como refugiado, así como los discursos y representaciones en torno a los solicitantes de asilo guardan un estrecho vínculo con las lecturas políticas que se construyen en destino en contextos particulares. Por ejemplo, una de las cuestiones latentes en Argentina en el año 2014, era la adopción el visado humanitario para personas de origen sirio en el marco del denominado Programa Siria.
} 
procesos políticos supone distanciarse de miradas que se centran solamente en "las motivaciones racionales" y poner el foco en "los significados culturales que determinadas conductas, valores y emociones tienen para los propios actores, quienes refieren y justifican sus acciones recurriendo a símbolos y mitos articulados en dispositivos rituales" (López Lara, 2005, p. 63).

\section{Consideraciones finales}

Los procesos de elegibilidad son centrales en la selección y categorización de las poblaciones, y son configurados en el marco de relaciones sociales y políticas que se encarnan en el desarrollo de los procedimientos de determinación de la condición de refugiado. He planteado la noción de ritual estatal de elegibilidad para analizar la forma en que operan los procedimientos en el marco de la elegibilidad, y los elementos clave en estos procesos instituyentes de la categoría de refugiado. Desde este lugar, los solicitantes de asilo evaluados atravesarían un proceso de institución (Bourdieu, 1997) que revela diferentes momentos y categorías posibles, apelando a estrategias para coincidir con los parámetros institucionales que supone tal nominación.

Por otro lado, el análisis de la elegibilidad como ritual estatal permite visibilizar las instancias por las que el solicitante debe atravesar, y las conductas y narrativas que debe desplegar, para ser instituido como refugiado por medio de la concesión del estatuto. Al mismo tiempo, pone en evidencia la forma en que opera la nominación estatal, por medio del portavoz autorizado, en este caso los agentes estatales, asignando una posición y a la vez significándola; es decir, la creación de la categoría recrea la misma estructura y poder estatal. El ritual es posible pensarlo no solamente como un rito que instituye a los sujetos, sino también a las instituciones; es decir, a la vez forman parte de aquello que construyen o va constituyendo a las mismas instituciones que los llevan adelante como organismos autorizados (Bourdieu, 1997).

Estos rituales de elegibilidad suponen diferentes instancias, partiendo del primer contacto con los guardianes del procedimiento, donde se presenta una verificación inicial; la presentación de la solicitud de asilo que plantea otra etapa de clasificación entre aquellos casos que ameritan procedimientos sumarios u ordinarios y un periodo liminal (Turner, 1988) de resolución. Hasta llegar a la situación de entrevista que se constituye en un evento central de dicho proceso, donde la emotividad tiene un lugar privilegiado.

Por otro lado, las implicancias sobre quién es instituido se revela en dos planos que tienen un efecto real en la persona, en tanto transforma la representación, y desde allí el comportamiento que los demás adoptan frente a esa persona consagrada bajo determinada categoría; y transforma la propia representación sobre sí mismo y el comportamiento en base a lo que se 
considera correspondiente a esa categoría asignada (Bourdieu, 1985, p. 80). Así, en el caso de las personas reconocidas como solicitantes o, más aún, como refugiadas no solo se pone de relieve la mirada de los otros desde la dimensión moral que interpela comportamientos solidarios y vinculados a la protección bajo la representación de víctimas, sino que también implica, para las personas instituidas como refugiados, colocar en un primer plano sus experiencias de sufrimiento y temor como parte del performance de la figura del refugiado reconocido, guardando en sus acciones los límites y obediencias a los presupuestos designados sobre el comportamiento como legitimo refugiado. A la par, esas implicancias sobre quienes atraviesan rituales de institución pueden verse encarnadas de manera similar a la investidura como funcionario de elegibilidad tanto en la representación como portavoz autorizado, como desde el comportamiento que de esta posición es esperado en su habilidad de discernimiento y categorización.

\section{Bibliografía}

ACNUR. La protección de los Refugiados y la Migración Mixta: el plan de los 10 Puntos en Acción. Ginebra, 2009.

ACNUR. Guía sobre el Derecho Internacional de los Refugiados. ACNUR/Unión Interparlamentária, 2001.

ACNUR. Parámetros para la Protección de los Refugiados en las Américas. 2000.

ÁLVAREZ, Jorge. Mejorar la Capacidad de Procesamiento de la Secretaria Ejecutiva de la Comisión Nacional para los Refugiados (Tesis de Maestría en Liderazgo y Administración Pública). España, Instituto Nacional de Administración Pública, 2015.

BOURDIEU, Pierre. ¿Qué significa hablar? Economía de los Intercambios Lingüísticos. Madrid: Ediciones Akal, 1985.

BOURDIEU, Pierre. Razones Prácticas. Sobre la Teoría de la Acción. Barcelona: Editorial Anagrama, 1997.

DOMENECH, Eduardo. Las migraciones son como el agua: Hacia la instauración de políticas de 'control con rostro humano'. Polis, n. 35, p. 1-21, 2013.

FASSIN, Didier. La razón Humanitaria. Una Historia Moral del Tiempo Presente. Buenos Aires: Prometeo Libros, 2016.

GIL ARAUJO, Sandra. Las argucias de la integración. Políticas migratorias, construcción nacional y cuestión social. Madrid: IEPALA, 2010.

KOBELINSKY, Carolina. ¿Emociones que corrigen la regla? El peso de las emociones en la Corte francesa del derecho al asilo. Papeles del CEIC, n. 91, marzo 2013.

LÓPEZ LARA, Álvaro. Los rituales y la Construcción Simbólica de la Política. Una Revisión de Enfoques. Sociológica, v. 19, n. 57, p. 61-92, 2005.

MALKKI, Liisa. National Geographic: The Rooting of Peoples and the Territorialization of National Identity among Scholars and Refugees. Cultural Anthropology, v. 7, n. 1, p. 24-44, 1992. 
Mc CALLUM, Stephanie. El refugiado hiperreal. Formas legítimas e ilegítimas de ser refugiado en la Argentina. Temas de Antropología y Migración, Dossier Asilo: entre el derecho y las prácticas, n. 4, p. 30-53, diciembre 2012.

NEJAMKIS, Lucila; ÁLVAREZ, Jorge. Migración y refugio en la Argentina: un análisis desde la legislación actual. Passagens. Revista Internacional de História Política e Cultura Jurídica, Rio de Janeiro, v. 4, n. 3, p. 445-463, 2012.

SAYAD, Abdelmalek. Estado, nación e inmigración. El orden nacional ante el desafío de la inmigración. Apuntes de Investigación del CECYP, n. 13, p. 101-116, 2008.

SAYAD, Abdelmalek. La doble ausencia. De las ilusiones del emigrado a los padecimientos del inmigrado. Barcelona: Anthropos, 2010.

SHORE, Chris; WRIGHT, Susan. Policy. A new field of Anthropology. In: SHORE, Chris; WRIGHT, Susan (eds.). Anthropology of Policy. Critical Perspectives on Governance and Power. London: Routledge, 1997.

TAUSSIG, Michael. Un gigante en convulsiones. El mundo humano como sistema nervioso en emergencia permanente. Barcelona: Gedisa, 1998.

TURNER, Victor. El proceso ritual. Estructura y antiestructura. Madrid: Taurus, 1988.

ZETTER, Roger. More labels, fewer refugees: Remaking the refugee label in an era of globalization. Journal of Refugee Studies, v. 20, n. 2, p. 172-192, 2007.

ZOLBERG, Aristide. The Formation of New States as a Refugee-Generating Process. Annals of the American Academy of Political and Social Science, v. 467, p. 24-38, 1983.

Recibido para publicación en 24.02.2018

Aceptado para publicación en 02.10.2018 Received for publication in February 24 ${ }^{\text {th }}, 2018$ Accepted for publication in October 02 $2^{\text {nd }}, 2018$

ISSN impresso 1980-8585

ISSN eletrônico 2237-9843

http://dx.doi.org/10.1590/1980-85852503880005409 